\title{
A COMPLETE CENSUS OF SILICATE FEATURES IN THE MID-INFRARED SPECTRA OF ACTIVE GALAXIES
}

\author{
E. Hatziminaoglou ${ }^{1}$, A. Hernán-Caballero ${ }^{2}$, A. Feltre $^{3}$, and N. Piñol Ferrer ${ }^{4}$ \\ ${ }^{1}$ ESO, Karl-Schwarzschild-Strasse 2, D-85748 Garching bei München, Germany \\ ${ }^{2}$ Instituto de Física de Cantabria, CSIC-UC, Avenida de los Castros s/n, E-39005, Santander, Spain \\ ${ }^{3}$ UPMC-CNRS, UMR7095, Institut d'Astrophysique de Paris, 98 bis boulevard Arago, F-75014 Paris, France \\ ${ }^{4}$ Department of Astronomy, Stockholm University, AlbaNova Center, SE-106 91 Stockholm, Sweden \\ Received 2014 November 25; accepted 2015 February 14; published 2015 April 22
}

\begin{abstract}
We present a comprehensive study of the silicate features at 9.7 and $18 \mu \mathrm{m}$ of a sample of almost 800 active galactic nuclei (AGNs) with available spectra from the Spitzer InfraRed Spectrograph (IRS). We measure the strength of the silicate feature at $9.7 \mu \mathrm{m}, S_{9.7}$, before and after subtracting the host galaxy emission from the IRS spectra. The number of type 1 and 2 AGNs with the feature in emission increases by 20 and $50 \%$, respectively, once the host galaxy is removed, while $35 \%$ of objects with this feature originally in absorption exhibit it in even deeper absorption. The peak of $S_{9.7}, \lambda_{\text {peak }}$, has a bimodal distribution when the feature is in emission, with about $65 \%$ of the cases showing $\lambda_{\text {peak }}>10.2 \mu \mathrm{m}$. Silicates can appear in emission in objects with mid-infrared (MIR) luminosity spanning over six orders of magnitude. The derived distributions of the strength of the silicate features at 9.7 and $18 \mu \mathrm{m}$ provide a solid test bed for modeling the dust distribution in AGNs. Clumpiness is needed in order to produce absorption features in unobscured AGNs and can also cause the silicates to be in absorption at $9.7 \mu \mathrm{m}$ and in emission at $18 \mu \mathrm{m}$ in type 1 sources. We find the "cosmic" silicates of Ossenkopf et al. to be more consistent with the observations than Draine's "astronomical" silicates. Finally, we discuss the possibility of a foreground absorber to explain the deep silicate absorption features in the MIR spectra of some type 2 AGNs.
\end{abstract}

Key words: galaxies: active - infrared: galaxies

\section{INTRODUCTION}

The InfraRed Spectrograph (IRS; Houck et al. 2004) on board the Spitzer Space Telescope provided, over a period of more than five years, low- and high-resolution mid-infrared (MIR) spectra from many thousands of galactic and extragalactic sources at wavelengths between 5 and $40 \mu \mathrm{m}$.

In active galactic nuclei (AGNs), the MIR emission is believed to be UV light reprocessed by the hot dust surrounding the AGN. The dust, often assumed to form a toroidal structure on parsec scales around the nucleus in the same plain as the accretion disk, is considered to be distributed either smoothly (e.g., Pier \& Krolik 1992; Granato \& Danese 1994; Fritz et al. 2006) or in clumps (e.g., Hönig et al. 2006; Nenkova et al. 2008). Dust is believed to consist of graphite and silicate grains, each leaving its unmistakable signature in the spectral energy distribution (SED) of AGNs, namely, the $\sim 1500 \mathrm{~K}$ blackbody-like rise of the MIR continuum of type 1 (unobscured) AGNs (e.g., Hatziminaoglou et al. 2005), corresponding to the sublimation temperature of graphites, and an absorption feature centered at $9.7 \mu \mathrm{m}$, long known to appear in type 2 (obscured) AGNs, attributed to silicate grains.

The uncertain observational evidence for silicate in the emission of type 1 AGNs (Clavel et al. 2000), however, has posed a problem for the Unified Scheme according to which the various types of AGNs can be explained by alignment effects between the central sources, the obscuring material (torus), and the observer (Antonucci 1993). The problem was finally solved when silicates were unambiguously observed in emission in the MIR spectra of many known AGNs with IRS (Hao et al. 2005; Siebenmorgen et al. 2005; Sturm et al. 2005; Buchanan et al. 2006; Shi et al. 2006).

Since then, various studies of the behavior of silicates have appeared. The very first such work, based on a few tens of
AGNs (e.g., Hao et al. 2007; Spoon et al. 2007; Wu et al. 2009), demonstrated that the silicate feature shows a wide range of diversity. In average spectra it varies with AGN type, ranging from moderate emission in bright quasars to almost no emission or slight absorption in Seyfert 1 galaxies to stronger absorption in Seyfert 2 galaxies. Meanwhile, sparse reports on the detection of silicates in emission (Mason et al. 2009; Nikutta et al. 2009) have shown the diversity of the dust properties, although some of them are rather rare. Several of the above observations also revealed a second silicate feature at $18 \mu \mathrm{m}$, as predicted by the models. The relative strength of the silicate features at 9.7 and $18 \mu \mathrm{m}$ has been put forward as a possible diagnostic of torus morphology (e.g., Thompson et al. 2009; Feltre et al. 2012) and chemistry (Sirocky et al. 2008).

In this paper, we assemble the largest sample of active galaxies with available IRS spectra ever composed (Section 2) with the aim of complementing and extending previous studies of the MIR characteristics of AGNs. To this end, we apply a new spectral decomposition technique to separate the nuclear emission from that of the host (Section 3). We then proceed with a thorough investigation of the behavior of the silicate feature at 9.7 and $18 \mu \mathrm{m}$ in the various AGN types (Section 4). Section 5 discusses our most important results and places them into a more general context.

\section{THE SAMPLE}

The Cornell AtlaS of Spitzer/IRS (CASSIS ${ }^{5}$; Lebouteiller et al. 2011) project has made available the reduced spectra of all of the sources observed with the low-resolution modules of IRS, a total of about 11,000 unique observations. Our master sample is derived from the CASSIS version 6 catalog, retaining

\footnotetext{
http://cassis.sirtf.com
} 
Table 1

Number of Objects per AGN Type

\begin{tabular}{lccc}
\hline \hline Type & $N_{\text {obj }}$ & Type & $N_{\text {obj }}$ \\
\hline Type 1 AGN & 363 & Sy1.2 & 24 \\
Type 2 AGN & 325 & Sy1 1.5 & 32 \\
$\ldots$ & $\ldots$ & Sy1.8 & 18 \\
SDSS quasars & 141 & Sy1.9 & 22 \\
\hline
\end{tabular}

each object that fulfills the following requirements: (i) has an identification in the NASA/IPAC Extragalactic Database ${ }^{6}$ (NED); (ii) has a robust (optical or infrared) spectroscopic redshift; (iii) the IRS spectrum fully covers the range between 6 and 13 micron rest frame; and (iv) the median signal-to-noise ratio $(\mathrm{S} / \mathrm{N})$ per pixel of the IRS spectrum is $>2$.

To verify requirement (i), we rely on the source crossidentification from CASSIS which matches the source coordinates with the NED and SIMBAD databases (Lebouteiller et al. 2011). For sources with no spectroscopic redshift in NED, we measure the redshift from the IRS spectrum using the template matching method presented in Hernán-Caballero (2012). The typical redshift uncertainty with this method is $\Delta z /(1+z) \sim 0.002$, which is well below the spectral resolution of our resampled spectra $(\Delta \lambda \lambda=0.005-0.02)$. The resampling also increases the minimum $\mathrm{S} / \mathrm{N}$ per resolution element from 2 to $>3$ (see Section 3 ).

Taking these criteria into account and removing duplicate entries, we end up with a list of 2299 extragalactic objects. Of these, 784 objects have an NED classification as type 1,2, or intermediate AGNs, and this is the sample we will be working with henceforth. AGNs of undefined type were left out of the sample. Among the type 1 AGNs are 141 quasars from the Sloan Digital Sky Survey (SDSS) Data Release 7 Quasar Catalog (Shen et al. 2011) which for a few specific purposes will be examined separately. The number of objects per subsample are shown in Table 1. The AGN sample is heterogeneous but nevertheless representative of the infrared (IR) AGN population.

\section{SPECTRAL DECOMPOSITION}

The observed MIR spectra of AGNs are affected by the presence of their host galaxies in two important ways. One way is the absorption or scattering of AGN emission by material (gas and dust) in the AGN line of sight (LOS). This so-called foreground absorption modulates the AGN spectrum with the multiplicative factor $e^{-\tau(\lambda)}$, where $\tau(\lambda)$ represents the optical depth at wavelength $\lambda$. It is not possible to distinguish foreground absorption from intrinsic AGN absorption (that is, the one produced in the AGN torus) from MIR data alone, since similar extinction laws are considered to apply to dust grains in the torus and the host. The amount of foreground extinction varies from source to source, but at MIR wavelengths it is expected to be mild in most sources, with the exception of some dusty starbursts and edge-on spiral galaxies. The other important effect on the AGN spectra is contamination from host galaxy emission that blends with the AGN spectrum. The importance of this background emission depends on the relative luminosities of the AGN and the host, and - crucially — on the spatial resolution of the spectroscopic

\footnotetext{
http://ned.ipac.caltech.edu
}

observations. Since the emission from the AGN is typically unresolved, an increase in the spatial resolution implies that a larger fraction of the host emission can be resolved away. In any case, the background emission represents an additive modification to the AGN spectrum.

The purpose of our spectral decomposition is to separate the AGN and host emissions in the integrated AGN+host spectra. If successful, this decomposition will allow us to study the AGN emission as if the host galaxy were resolved away. To this end, we employ the decomposition method presented in Hernán-Caballero et al. (2015). Their method relies on the large number of high-quality spectra in CASSIS to reproduce the spectra of composite sources as a linear combination of three CASSIS spectra, each selected from subsamples of sources whose mid-IR emission is completely dominated by the AGN, star formation, or stellar population. We select these "single-spectral-component" templates as follows.

For the "stellar" templates, we select 19 local elliptical and S0 galaxies. To ensure that they have negligible star formation, we require the polycyclic aromatic hydrocarbon ( $\mathrm{PAH}$ ) bands to be very weak or absent with equivalent widths for the $6.2 \mu \mathrm{m}$ $\left(\mathrm{EW}_{62}\right)$ and $11.3 \mu \mathrm{m}\left(\mathrm{EW}_{113}\right)$ PAH features $<0.02 \mu \mathrm{m}$. We also check to ensure that the IRS spectra have a blue stellar-like MIR continuum and that the sources are not classified as AGNs in NED. The 54 star-forming templates ("PAH" templates) are IRS spectra of normal star-forming and starburst galaxies at redshifts up to $z=0.14$. We make sure that these sources do not have significant stellar contributions to their MIR spectra by requiring both high EWs of the $\mathrm{PAH}$ features $\left(\mathrm{EW}_{62}>1.0 \mu \mathrm{m}\right.$ and $\left.\mathrm{EW}_{113}>1.0 \mu \mathrm{m}\right)$ and a very weak continuum at $5 \mu \mathrm{m}$. We also verify that they are not classified as AGNs in NED. Finally, the 147 "AGN" templates are IRS spectra of sources classified in the optical as quasars, Seyfert galaxies, LINERs, and blazars. We also include a variety of optically obscured AGNs and radio galaxies. The templates include sources at redshifts from $z=0.002$ to $z=1.4$ and cover several orders of magnitude in bolometric luminosity. We ensure that the AGN templates do not contain any significant emission from the host galaxy by requiring the $\mathrm{PAH}$ features to be extremely weak or absent $\left(\mathrm{EW}_{62}<0.02 \mu \mathrm{m}\right.$ and $\mathrm{EW}_{113}<$ $0.02 \mu \mathrm{m})$.

Because the AGN and PAH templates are real spectra, each of them already includes some amount of foreground extinction built in. Therefore, we rely on the large number of AGN and $\mathrm{PAH}$ templates to reproduce the diversity of observed spectra arising from different levels of foreground extinction as well as source to source variation in the intrinsic AGN and host spectra. This approach has the advantage of not depending on assumptions about the-unobservable-intrinsic AGN spectrum or the extinction law. Obtaining a good fit using this decomposition method requires finding an AGN template with the appropriate level of foreground absorption. This can be problematic for sources with very deep absorption features, since few pure-AGN spectra have such features. Therefore, decomposition results for sources with deep absorption features have larger residuals and uncertainties.

We separate the AGN sample into two groups depending on spectral coverage: those objects with silicate features observed at both 9.7 and at $18 \mu \mathrm{m}$ in their full extent, and those for which only the feature at $9.7 \mu \mathrm{m}$ is covered. For sources in the first group, we fit the spectral range between the 5.2 and $22 \mu \mathrm{m}$ rest frame, while for those in the second we fit only the 5.2 to 
$15.8 \mu \mathrm{m}$ range. We resample both the spectra and templates to a common wavelength grid with a uniform wavelength resolution of $\Delta \lambda=0.1 \mu \mathrm{m}$. This increases the $\mathrm{S} / \mathrm{N}$ per resolution element by $\sim 60 \%$ on average while still allowing us to resolve important features such as the PAH bands. For every galaxy in the sample, we try performing spectral decompositions using every possible combination of stellar template, $\mathrm{PAH}$ template, and AGN template. The best-fitting model is that which produces the absolute minimum $\chi^{2}$. However, to calculate expected values for observables (e.g., the luminosity of the AGN component or the strength of the silicate feature) and their uncertainties, we use the full probability distribution functions (PDFs) calculated with the "max" method described in Noll et al. (2009; see Section 2 in Hernán-Caballero et al. 2015 for details). The method also yields for each object the fractional contribution of each of the three components to the total luminosity in the wavelength range of interest.

Thanks to the use of large sets of real spectra as templates, our decomposition method manages to reproduce the MIR spectrum of composite sources with unprecedented accuracy (see Figure 1). Typical $\chi^{2}$ values are lower than two, indicating that residuals in the model fits are dominated by noise in both the spectra and templates for most sources.

\section{THE SILICATE FEATURES}

The silicate features at 9.7 and $18 \mu \mathrm{m}$ observed in the IR spectra of AGNs are believed to arise from the inner, hotter parts of the torus or the hot, illuminated side of the clumps. We define the strength of the silicate feature following Pier \& Krolik (1992):

$$
S_{\lambda}=\ln \frac{F\left(\lambda_{\text {peak }}\right)}{F_{\mathrm{c}}\left(\lambda_{\text {peak }}\right)}
$$

where $F\left(\lambda_{\text {peak }}\right)$ and $F_{\mathrm{c}}\left(\lambda_{\text {peak }}\right)$ are the flux densities of the spectrum and the underlying continuum at the peak wavelength of the features, $\lambda_{\text {peak }}$. A negative (positive) value indicates a feature in absorption (emission).

\subsection{The Silicate Feature at 9.7 Micron}

The top panel of Figure 2 shows the distribution of the strength of the silicate feature at $9.7 \mu \mathrm{m}$ as measured in the original IRS spectra, $S_{9.7 \text { tot }}$, for type 1 and type 2 AGNs (the 96 AGNs of intermediate type are not included here and will be discussed separately).

As has already been observationally established in the past $10 \mathrm{yr}, S_{9.7 \text { tot }}$ takes a wide range of values (e.g., Hao et al. 2007; Levenson et al. 2007; Spoon et al. 2007; Wu et al. 2009, just to name a few). The average spectra of type 1 AGNs exhibit this feature in weak to moderate emission (see, e.g., Hao et al. 2007; Wu et al. 2009), while those of type 2 AGNs present this feature in absorption (Sturm et al. 2006; Schweitzer et al. 2008; Mason et al. 2009; Hernán-Caballero \& Hatziminaoglou 2011). Individually, however, type 1 and type 2 AGNs can show silicates in absorption and emission, respectively. In our sample of 698 type 1 and 2 AGNs, 35\% of the type 1 AGNs present this feature in absorption and about $15 \%$ of the type 2 AGNs show this feature in emission.

At the same time, when in emission, the peak of the feature is often shifted to wavelengths longer than $9.7 \mu \mathrm{m}$ in the rest frame, while the shift affects the feature much less when in absorption, as already reported by, e.g., Shi et al. (2014). Figure 3 shows the distribution of the shift, $\Delta \lambda_{\text {peak }}=\lambda_{\text {peak }}-9.7 \mu \mathrm{m}$, as a function of the fractional contribution of the AGN to the luminosity in the range between 5 and $15 \mu \mathrm{m}, f_{\mathrm{AGN}}$, for type 1 and type 2 objects (filled and open symbols, respectively), color-coded by $S_{9.7}$ tot. Looking at the sample as a whole, $65 \%(20 \%)$ of the objects with silicates in emission have their $\lambda_{\text {peak }}>10.2 \mu \mathrm{m}\left(\lambda_{\text {peak }}>10.6 \mu \mathrm{m}\right)$, while the fraction of objects with the same amount of shift among the AGNs with silicates in absorption is less than $3 \%$. The shift to longer wavelenghts is largely associated with a silicate feature in emission, which in turn only occurs in strongly AGN-dominated spectra.

\subsection{Removing the Effects of the Host}

As $S_{9.7 \text { tot }}$ are measured in the original IRS spectra, we expect the derived values to be contaminated by emission from the host galaxy for all objects except those for which the AGN completely dominates the MIR emission. The top panel of Figure 4 shows $S_{9.7 \text { tot }}$ as a function of $f_{\mathrm{AGN}}$. The filled and open symbols correspond to type 1 and type 2 AGNs, respectively. Error bars for $S_{9.7 \text { tot }}$ are shown in this plot but they will not be repeated in following figures, in order to keep the plots the least crowded as possible. What we see here is that as the contribution of the host becomes more important (i.e., as $f_{\text {AGN }}$ decreases), the strength of the silicate feature decreases with only a few objects exhibiting $S_{9.7 \text { tot }}>0.0$ for $f_{\mathrm{AGN}}<0.7$. The dashed line shows the (weak) trend for the full sample with a linear correlation coefficient of $r=0.45$. The trend, however, is driven by type 1 objects (filled symbols and corresponding solid line) due to contamination from the emission of the host galaxy.

In order to see how the silicates behave in the vicinity of the nucleus, we need to remove the contribution of the host galaxy, applying the spectral decomposition procedure described in Section 3. The distribution of the strength of the silicate feature on the host-subtracted spectrum, $S_{9.7 \mathrm{AGN}}$, is shown in the

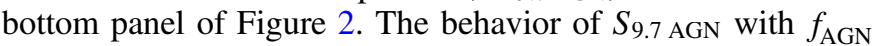
shown in the lower panel of Figure 4 differs from that of $S_{9.7}$ tot in that there is now no correlation between the two quantities, confirming that the correlation was indeed due to contamination from the emission of the host.

A direct comparison of the two measurements of the silicate feature at $9.7 \mu \mathrm{m}$ is shown in Figure 5, color-coded by the value of $f_{\mathrm{AGN}}$. Objects with MIR emission completely dominated by the AGN (light-color symbols) are not affected by the subtraction of the host (they lie on or very near the $1: 1$ line). However, as the contribution of the host galaxy becomes more important, i.e., as $f_{\mathrm{AGN}}$ decreases (symbols become darker in Figure 5), the points deviate more and more from the $1: 1$ line.

By subtracting the emission of the galaxy, the number of type 1 AGNs with silicates in emission increases by $20 \%$ reaching $80 \%$ of all type 1 AGNs, while the number of type 2 AGNs with the feature in emission doubles reaching a total of $25 \%$. At the same time, $35 \%$ of both type 1 and type 2 AGNs with this feature in absorption exhibit the feature in even deeper absorption once the emission from the host is removed. This happens because AGNs with a silicate feature in deeper absorption than that of the surrounding host have their silicate feature "refilled" in the integrated spectrum. 


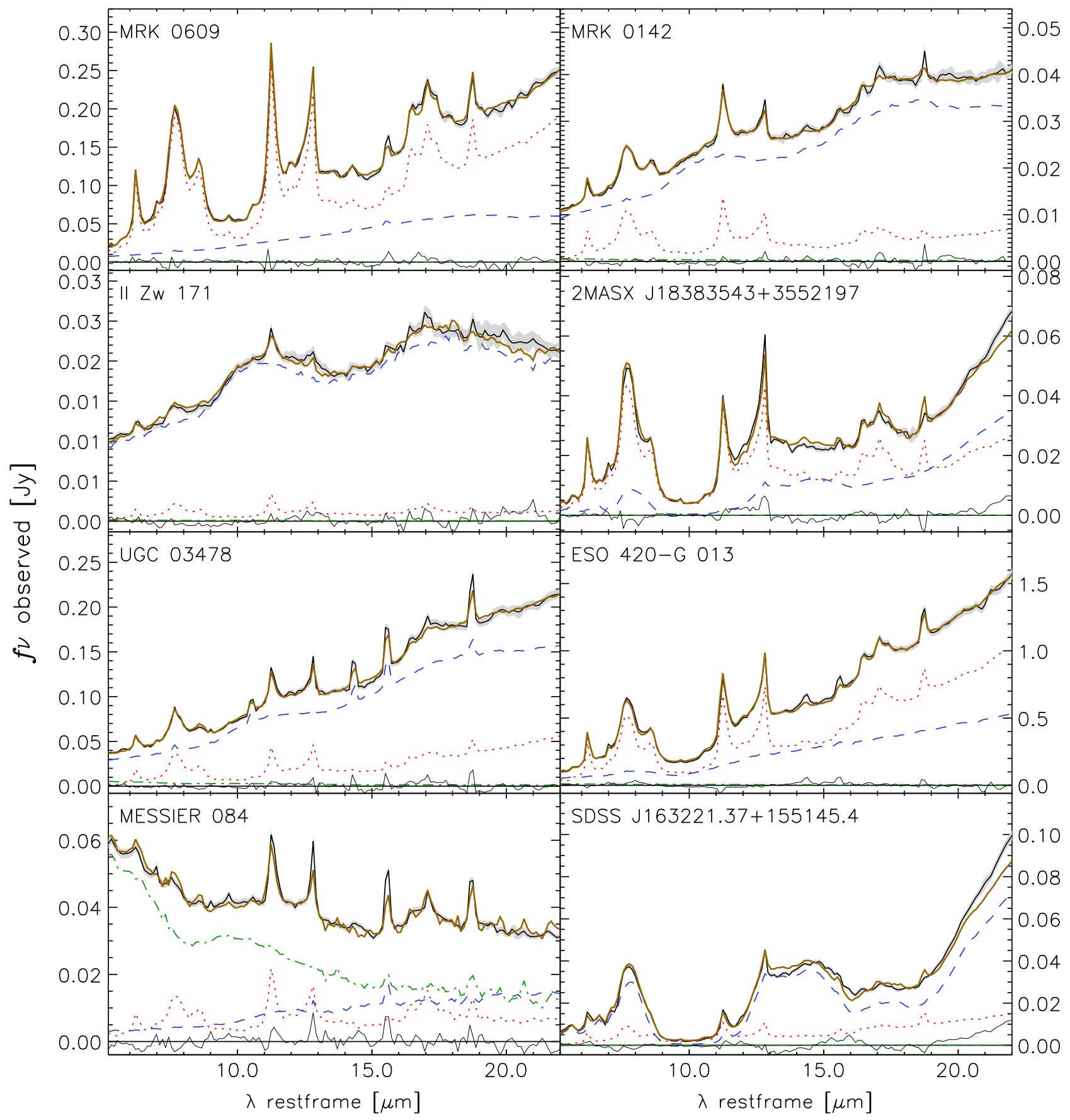

Figure 1. Examples of best-fitting decomposition models for the IRS spectra. The black solid line with gray shading represents the IRS spectrum (resampled at $\Delta \lambda$ $=0.1 \mu \mathrm{m}$ resolution) and its $1 \sigma$ uncertainty (photometric errors only). The dotted, dashed, and dot-dashed lines represent, respectively, the PAH, AGN, and stellar components of the best-fitting model, shown in yellow. The thin solid line at the bottom of each plot represents the residual (spectrum-model).

In order to check whether $S_{9.7 \mathrm{AGN}}$ is affected by the AGN luminosity, as proposed by, e.g., Maiolino et al. (2007), we have to rely on the luminosity at $7 \mu \mathrm{m}, L_{7}$, that spans over six orders of magnitude in our sample. MIR luminosity in AGNs has, in fact, been shown to tightly correlate with X-ray luminosity (see e.g., Lutz et al. 2004; Horst et al. 2008; Mateos et al. 2015); however, the ratio of the MIR to the bolometric luminosity depends on the column density along the LOS (Figure 21 in Hatziminaoglou et al. 2009). Figure 6 shows $S_{9.7 \mathrm{AGN}}$ as a function of $L_{7}$ measured in the galaxy-subtracted spectrum with the points colored based on redshift. There is clearly no dependence of $S$ $9.7 \mathrm{AGN}$ on $L_{7}$ and the features can be in emission $\left(S_{9.7}>0.0\right)$ even in the faintest AGNs. Deep silicate features $\left(S_{9.7}<-2\right)$ are only found at intermediate luminosities (or redshifts). A deeply obscured but low-luminosity AGN would be overwhelmed by the emission of its host, which would "refill" the silicate feature. The lack of deep silicates in objects with very high IR luminosities and/or high redshifts, on the other hand, suggests a selection effect, as they might be too faint in the optical for a reliable identification. 

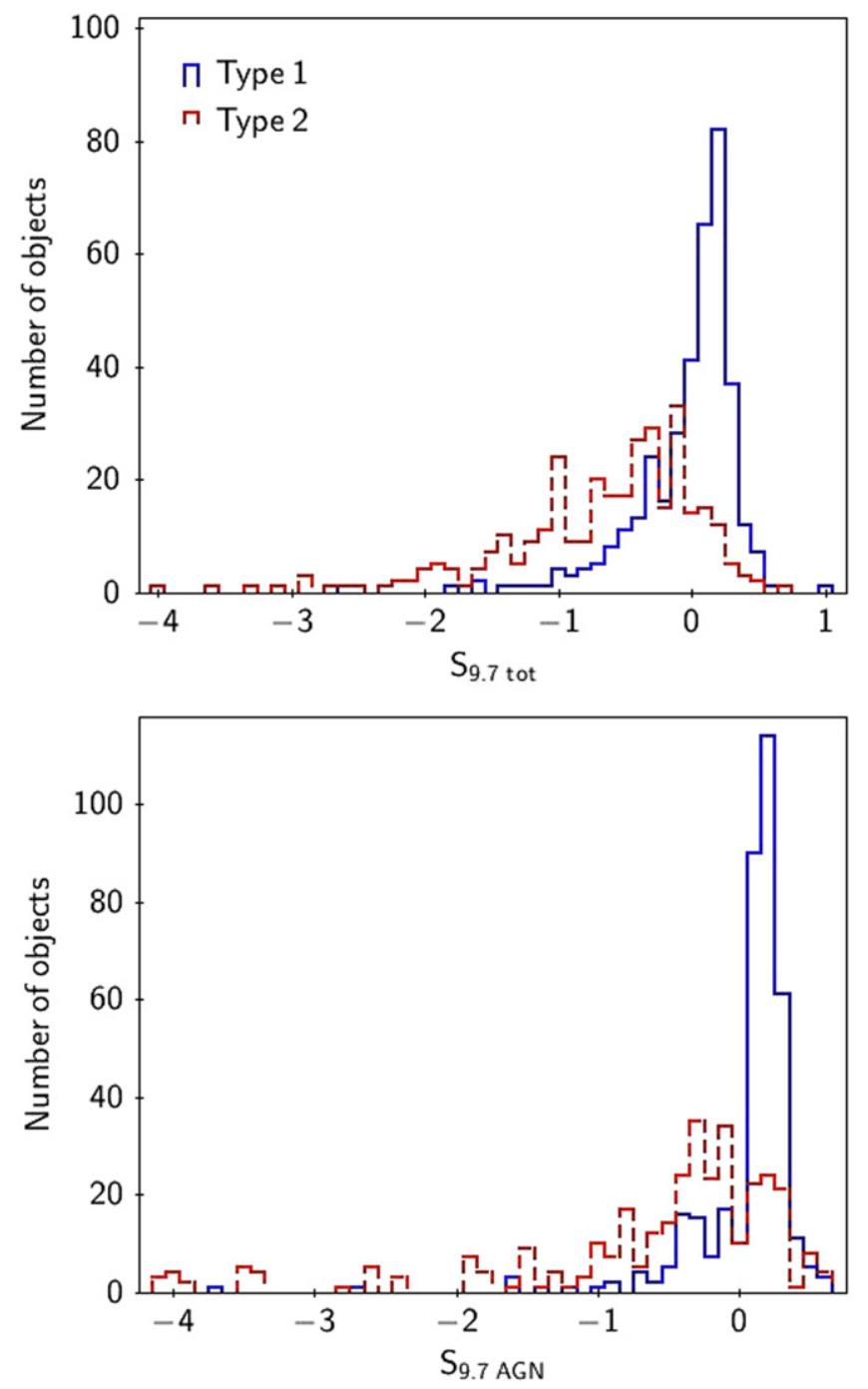

Figure 2. $S_{9.7}$ distribution for type 1 (blue solid histogram) and type 2 (red dashed histogram) before (top panel) and after (bottom panel) the subtraction of the host galaxy.

\subsubsection{AGNs of Intermediate Types}

Among the 784 AGNs of our sample, 96 have an NED classification of intermediate-type Seyfert galaxies. The number of objects per type (comparable in all four subsamples) is shown in Table 1. Figure 7 shows the distribution of $S_{9.7}$ AGN for the four subsamples.

The mean average $S_{9.7 \text { AGN }}$ decreases from Sy1.2 toward later types with Sy 1.2, 1.5, and 1.8 showing on average the silicate feature in very weak or no emission, while Sy1.9 has a negative average value. Additionally, as we move to later intermediate types, the dominance of the AGN component to the MIR emission decreases; while $96 \%$ of Sy1.2 objects have $f_{\mathrm{AGN}}>0.7$, the fraction drops to $55 \%$ for Sy1.9 s. This is in agreement with Deo et al. (2007), who found the MIR spectra of Sy1.8 and Sy1.9 to be dominated by starburst features (PAH). The mean values of $S_{9.7 \text { AGN }}$ and the fraction of objects with $f_{\mathrm{AGN}}>0.7$ for each intermediate type are shown in Table 2. Note that the shift toward lower values of $S_{9.7 \text { AGN }}$ when moving toward later Seyfert types persists even when only objects with $f_{\mathrm{AGN}}>0.7$ are considered, as seen in the right-most column of Table 2 . Finally, the $\lambda_{\text {peak }}$ of intermediate

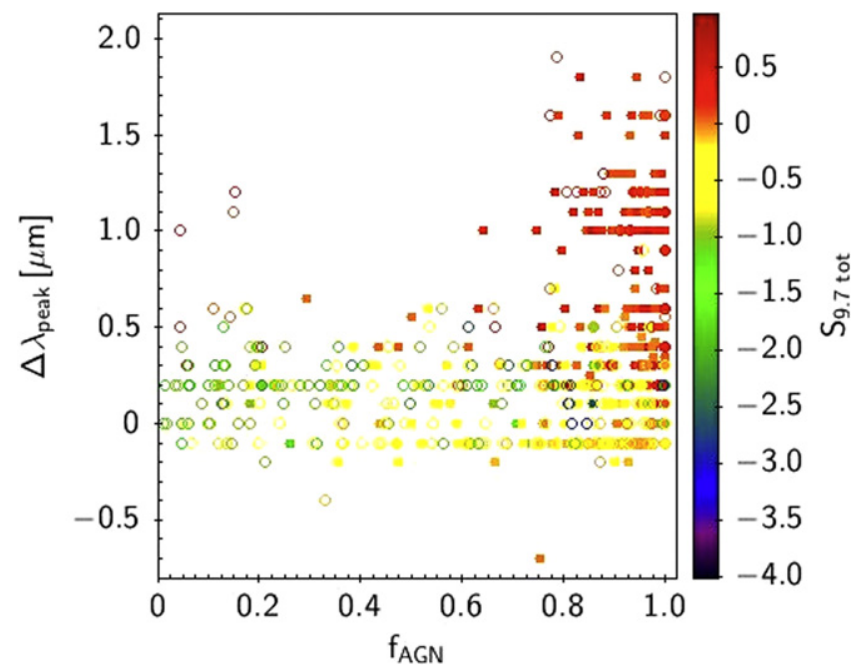

Figure 3. $\Delta \lambda_{\text {peak }}$ as a function of $f_{\mathrm{AGN}}$ for type 1 and type 2 objects (filled and open symbols, respectively). The symbols are color-coded based on the value

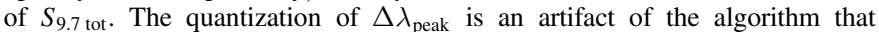
measures $\lambda_{\text {peak }}$.

Seyfert types shows the same behavior as for the rest of the AGN sample, i.e., as described in Section 4.1.

\subsubsection{SDSS Quasars}

Of the 784 AGNs, 141 are spectroscopically confirmed SDSS quasars for which estimates of the mass of the central black hole, $M_{\mathrm{BH}}$, derived from emission line measurements as well as bolometric luminosities, $L_{\mathrm{bol}}$, derived from fitting techniques are available (Shen et al. 2011). Maiolino et al. (2007) reported an increase of $S_{9.7}$ with increasing $M_{\mathrm{BH}}$ from low-luminosity, low-redshift type 1 AGNs to high-luminosity, high-redshift quasars. Thompson et al. (2009), on the other hand, found no trend with luminosity. $M_{\mathrm{BH}}$ for the 141 SDSS quasars in question spans the range $\left[10^{7.3}-10^{10} M_{\odot}\right]$, i.e., almost identical to that of the Maiolino et al. (2007) sample, but we do not find any correlation of $S_{9.7 \mathrm{AGN}}$ with $M_{\mathrm{BH}}$, as shown in Figure 8. Note, however, that the bolometric luminosities of the SDSS quasars of our sample are all above $8.2 \times 10^{10} L_{\odot}\left(10^{44.5} \mathrm{erg} \mathrm{s}^{-1}\right)$, i.e., the two samples are not directly comparable. In the quasar subsample, $S_{9.7 \text { AGN }}$ and $L_{\text {bol }}$ are completely uncorrelated (see color-coding in Figure 8), in agreement with Thompson et al. (2009).

\subsection{The Silicate Feature at 18 Micron}

The silicate feature at $18 \mu \mathrm{m}$, although predicted by models and observed in many of the low-to-intermediate redshift AGNs ( $z$ typically $\leqslant 0.5$; e.g., Hao et al. 2005; Sirocky et al. 2008; Thompson et al. 2009), has received less attention than its lower wavelength counterpart as its measurement presents a greater challenge. For one, it often overlaps with the MIR bump of the AGN SED (e.g., Prieto et al. 2010). Also, the steep mid-to-far-IR emission from the host implies that it contributes a higher fraction to the continuum emission at these longer wavelengths, making the measurement of the feature a tedious job. Following the procedure described in Section 3 but extending the wavelength range to $22 \mu \mathrm{m}$ rest frame, we perform spectral decompositions and measure the strength of the $18 \mu \mathrm{m}$ silicate feature in the AGN component, $S_{18 \text { AGN }}$, for 


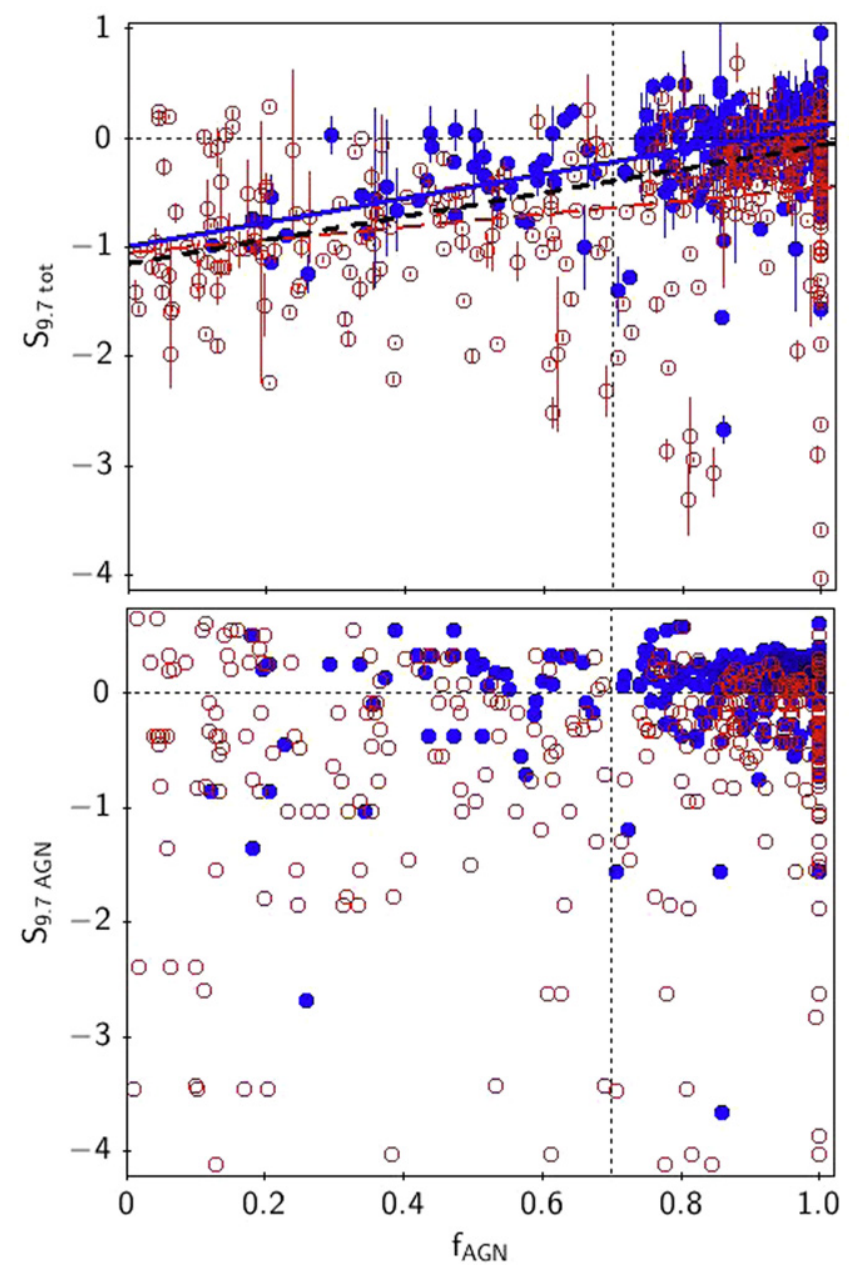

Figure 4. $S_{9.7}$ as a function of $f_{\mathrm{AGN}}$ before (upper panel) and after (lower panel) subtraction of the host galaxy. Filled and open circles denote type 1 and type 2 AGNs, respectively. The thin dashed lines show $S_{9.7}=0.0$ and $f_{\mathrm{AGN}}=0.7$, the thick dashed line shows the weak $(r=0.45)$ linear correlation of the full sample, and the solid and long-dashed lines show the correlations for the type $1(r=0.51)$ and type $2(r=0.26)$ objects, respectively.

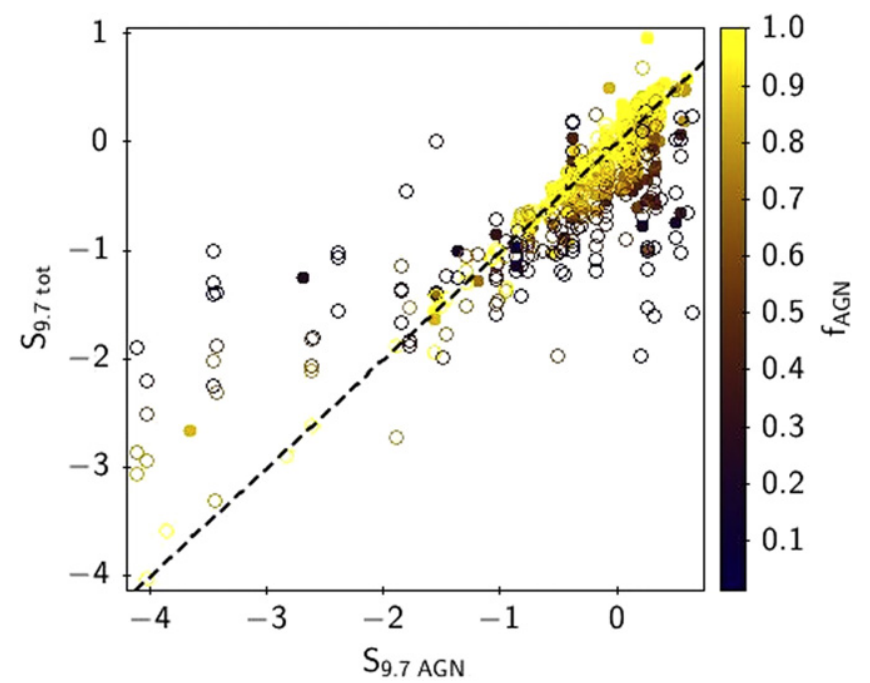

Figure 5. $S_{9.7 \text { tot }}$ vs. $S_{9.7 \mathrm{AGN}}$ color-coded based on $f_{\mathrm{AGN}}$. Filled and open symbols denote type 1 and type 2 AGNs, respectively.

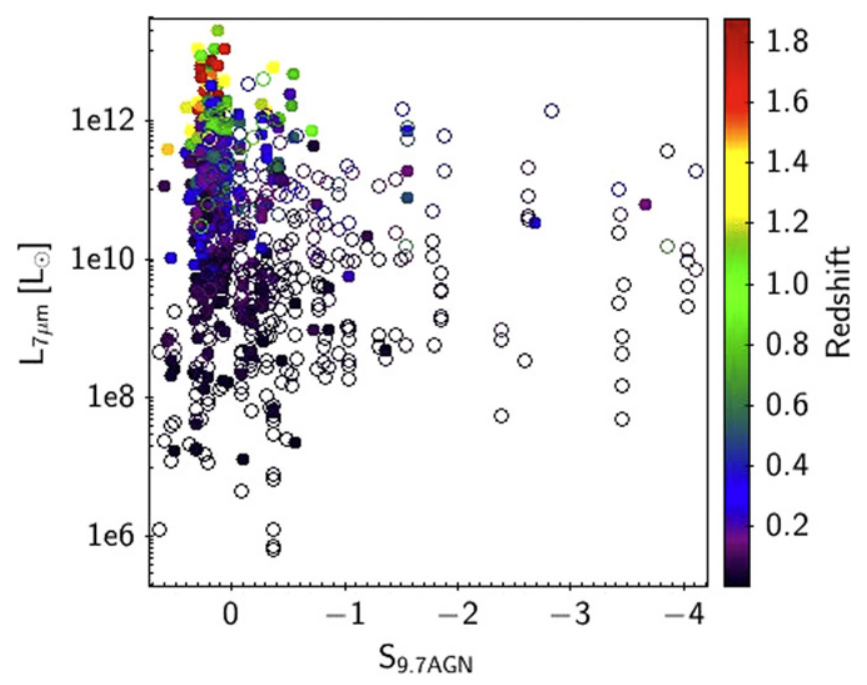

Figure 6. Relation between $S_{9.7 \text { AGN }}$ and $L_{7}$ with points color-coded based on their redshift. The filled and open circles correspond to type 1 and type 2 AGNs, respectively.

the 631 AGNs with adequate wavelength coverage. Figure 9 shows the distribution of $S_{18 \mathrm{AGN}}$ for type 1 (blue solid histograms) and type 2 (red dashed histograms) AGNs.

While at least as prominent as its counterpart at $9.7 \mu \mathrm{m}$ in emission, the feature only reaches moderate depths in absorption. Furthermore, more than $50 \%$ of type 2 AGNs exhibit this feature in emission while only $10 \%$ of type 1 AGNs have it in absorption. Comparing these numbers with those for $S_{9.7 \text { AGN }}$ it becomes obvious that the feature can be in absorption at $9.7 \mu \mathrm{m}$ while still in emission at $18 \mu \mathrm{m}$. We will return to this point in Section 5 .

\section{DISCUSSION AND CONCLUSIONS}

Using the largest sample of AGNs with MIR spectroscopy ever assembled, we quantify, for the first time, the effects of the emission of the host galaxy on the behavior of the silicate features, a tracer of the properties of the hot dust in the torus. We rely on the classification provided by NED in order to call an object an "AGN" as well as for the classification of AGNs into types 1 and 2. The sample includes a variety of AGNs, from objects where the AGN completely dominates the MIR emission $\left(f_{\mathrm{AGN}} \sim 1\right)$ to $\mathrm{AGN}$ whose MIR emission is almost entirely dominated by star formation $\left(f_{\mathrm{AGN}} \ll 1\right)$. We find the fraction of objects with a strong contamination from the galaxy $\left(f_{\mathrm{AGN}}<0.7\right)$ to be much higher among type 2 AGNs $(43 \%)$ than among type 1 AGNs (12\%).

The emission of the host affects the behavior of the silicate features. Broadly speaking, the strength of the silicate feature at $9.7 \mu \mathrm{m}$ is a measure of the optical depth, $\tau_{9.7}$, along the LOS, and goes from emission to absorption as $\tau_{9.7}$ increases (see, e.g., Fritz et al. 2006, Figure 9). In this simple picture, type 1 and type 2 AGNs should show the feature in emission and absorption, respectively. Our study, however, shows that type 1 (type 2) AGNs with the feature in absorption (emission) are very common, even after subtracting the contribution of the host galaxy. The number of type 1 and 2 AGNs with the feature in emission increases by 20 and $50 \%$, respectively, once the host galaxy is removed, while $35 \%$ of objects with the feature originally in absorption exhibit it in even deeper absorption 


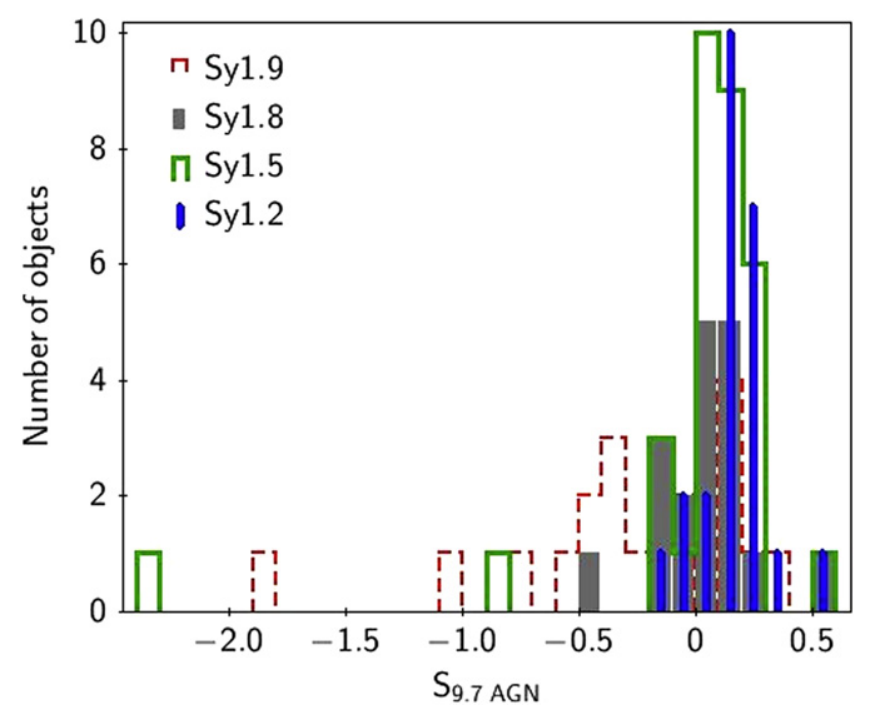

Figure 7. Distribution of $S_{9.7 \mathrm{AGN}}$ for the four intermediate types of Seyfert galaxies.

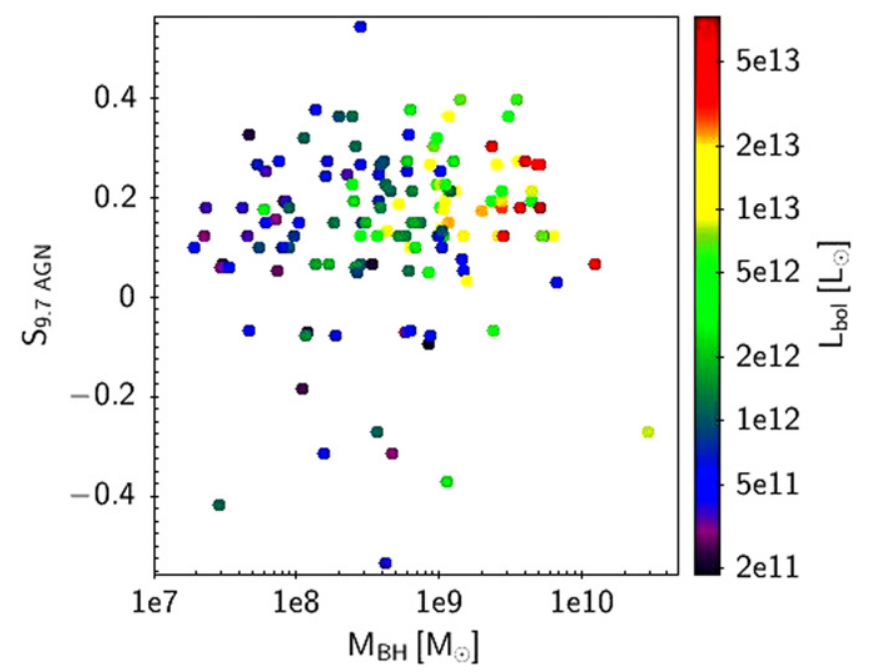

Figure 8. $S_{9.7 \mathrm{AGN}}$ as a function of $M_{\mathrm{BH}}$ for the 141 SDSS quasars of the sample. The points are color-coded by $L_{\text {bol }}$.

after subtraction of the host. This means that the combined spectrum exhibits an $S_{9.7 \text { tot }}$ intermediate between $S_{9.7 \text { AGN }}$ and that of the host. The host galaxy nearly always shows mild silicate absorption, as the power sources (stars) are well mixed with the absorbing dust, and therefore the strength of the silicate feature does not correlate with the optical depth of the gas and dust along the LOS. Consequently, contamination from the host increases the depth of the absorption if the feature intrinsic to the AGN is in emission or mild absorption, but decreases the depth (i.e., fills the gap) if the AGN has a deeper feature than the host.

$S_{9.7 \text { tot }}$ is scarcely ever shown in emission when the MIR emission is strongly contaminated by the host galaxy $\left(f_{\mathrm{AGN}}<0.7\right)$, with those objects exhibiting the feature in deeper absorption on average than their AGN-dominated counterparts. $S_{9.7 \mathrm{AGN}}$, on the other hand, shows no dependency on $f_{\mathrm{AGN}}$ and appears from moderate emission to quite deep absorption with all possibilities in between. The lack of correlation between $S_{9.7 \mathrm{AGN}}$ and $f_{\mathrm{AGN}}$, two quantities that are

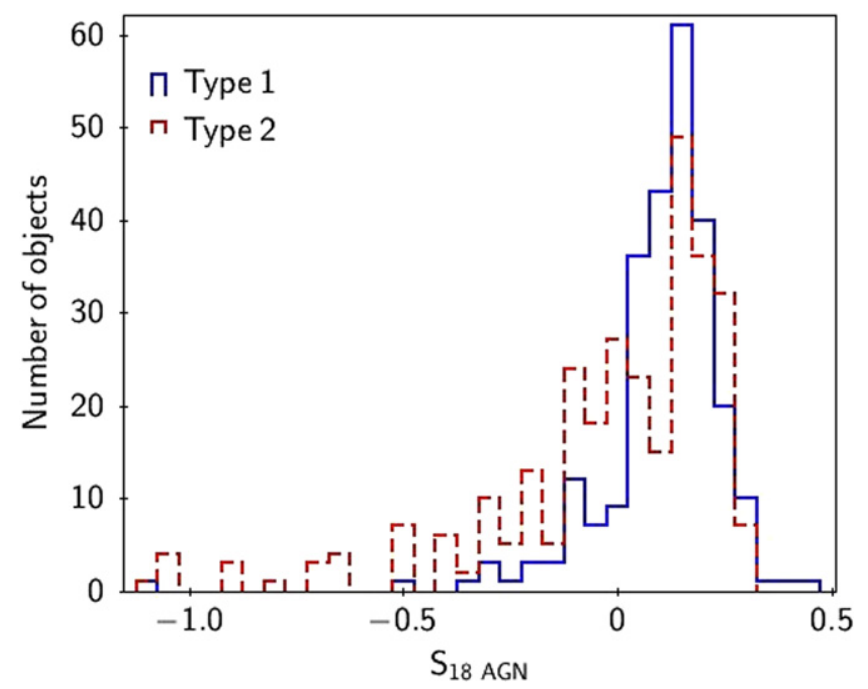

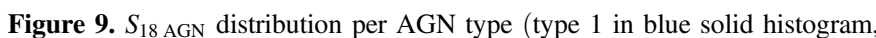
type 2 in red dashed histogram).

Table 2

Mean Values and Standard Deviations of $S_{9.7}$ for Each Intermediate AGN Type, Fraction of Objects with $f_{\mathrm{AGN}}>0.7$, and $S_{9.7}$ for that Fraction

\begin{tabular}{lccr}
\hline \hline Type & $\left\langle S_{9.7 \text { tot }}\right\rangle$ & $f_{\mathrm{AGN}}>0.7$ & \multicolumn{1}{c}{$\left\langle S_{9.7 \text { tot }}^{f_{\mathrm{AN}}>0.7}\right\rangle$} \\
\hline Sy1.2 & $0.175 \pm 0.13$ & $96 \%$ & $0.160 \pm 0.14$ \\
Sy1.5 & $0.043 \pm 0.46$ & $90 \%$ & $0.087 \pm 0.22$ \\
Sy1.8 & $0.022 \pm 0.22$ & $61 \%$ & $0.059 \pm 0.11$ \\
Sy1.9 & $-0.305 \pm 0.49$ & $55 \%$ & $-0.227 \pm 0.35$ \\
\hline
\end{tabular}

physically unrelated, indicates that the decomposition mechanism successfully removes the most important part of the host galaxy emission, allowing for an almost unbiased silicate measurement of the silicate feature in the vicinity of the nucleus.

We have also addressed the issue of the observed shift of the silicate feature at $9.7 \mu \mathrm{m}$ to longer wavelengths with respect to the nominal peak at $9.7 \mu \mathrm{m}$. Our decomposition method does not allow a reliable estimate of $\lambda_{\text {peak }}$ on the galaxy-subtracted spectra. We therefore rely on the measurement carried out on the original IRS spectra. We find the largest shifts $\left(\lambda_{\text {peak }}>10.2 \mu \mathrm{m}\right)$ to appear only in objects with an important AGN component $\left(f_{\mathrm{AGN}}>0.7\right)$ with the feature in emission, regardless of their type. When the feature is in absorption, and again irrespective of the type, it appears at or near its nominal wavelength $\left(\lambda_{\text {peak }}<10.2 \mu \mathrm{m}\right)$. Since its discovery, various scenarios have been proposed in order to explain this shift, such as the presence of porous dust ( $\mathrm{Li}$ et al. 2008; Smith et al. 2010), the presence of different dust species (Markwick-Kemper et al. 2007), or radiative transfer effects (Nikutta et al. 2009). Shi et al. (2014), however, posit that this is not a radiative transfer effect but rather the effect of direct exposure of the silicates to nuclear radiation which modifies the size or the chemical composition of the grains, more along the lines of Smith et al. (2010).

\subsection{Dust Distribution Inside and Outside the Torus}

$S_{9.7 \mathrm{AGN}}$ is seen in only moderate emission or slight absorption in most type 1 AGNs, behavior that traditionally favors a clumpy morphology, both because smooth models 
often predict silicates in stronger emission than observed and because, with the exception of a couple of smooth model parameter combinations, only clumpiness can explain the feature in absorption in unobscured AGNs. However, and even though not producing the feature in absorption, a continuous dust distribution can also give rise to silicates in only weak emission for a large variety of parameters, as shown in Figure 4 of Feltre et al. (2012). Furthermore, Sirocky et al. (2008) showed that the use of the Ossenkopf et al. (1992) dust absorption and scattering coefficients result in considerably less prominent silicate emission features compared to other dust models like Draine (2003). We therefore interpret the behavior

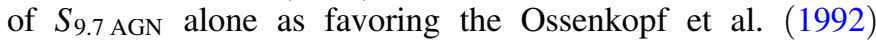
silicates over the Draine (2003) silicates, but this property alone cannot provide much insight into the morphology of the dust.

The transition of the mean values of $S_{9.7}$ from weak emission to absorption from Sy1.2 to Sy1.9 can be explained by either dust morphology: in a smooth medium, it can be attributed to an increase of the inclination (as measured from the poles), and hence the intervening material with the silicate feature at $9.7 \mu \mathrm{m}$ that arises from the inner, hotter parts of the torus being increasingly blocked by the bulk of the dust as the viewing angle increases. In a clumpy medium, on the other hand, this could simply be attributed to different levels of obscuration along the LOS, independent of the orientation.

The combined strength of the silicate features at 9.7 and $18 \mu \mathrm{m}$ is sensitive to the chemistry and morphology of the dust surrounding the AGN, i.e., the torus (Sirocky et al. 2008; Thompson et al. 2009; Feltre et al. 2012). To test this, we created three grids of models, two clumpy and a smooth, following Nenkova et al. (2008) and Feltre et al. (2012), respectively. All three sets of models share the same primary source, described in Nenkova et al. (2008). One of the clumpy grids was created using the silicate absorption and scattering coefficients from Draine (2003), while the other clumpy and smooth grids were created using the Ossenkopf et al. (1992) silicates. The models have been created to have matched parameters, as defined in Feltre et al. (2012), i.e., each of the smooth models in the grid has an equivalent model (in terms of geometrical properties) in the clumpy grid. The parameter space explored by the model grids is briefly described in the Appendix.

Figure 10 shows the distributions of $S_{18 \mathrm{AGN}}$ and $S_{9.7 \mathrm{AGN}}$, compared to model predictions. The top (bottom) panel shows type 1 (type 2) AGNs (in blue). Smooth models are shown in green, and clumpy models using the Ossenkopf et al. (1992) and Draine (2003) silicates are shown in gray and pink, respectively. The spread of observed data points indicates the variety of torus geometries in nature in terms of size, shape, and optical depths.

As previously noted by other authors, the so-called "astronomical" silicates (Draine 2003) produce stronger emission features at $9.7 \mu \mathrm{m}$ and a wider range of $S_{18 \mathrm{AGN}} / S$ 9.7 AGN than what is observed compared to the Ossenkopf et al. (1992) silicates. The bulk of type 1 objects lie in the region of overlap between smooth and clumpy models. Overall, models reproduce in a better fashion the silicates in type 2 AGNs. However, both smooth and clumpy models, albeit covering a large parameter space, fail to reproduce the deep absorption features seen in type 1 AGNs (lower left quadrant in top panel of Figure 10). At the same time, as already suggested by
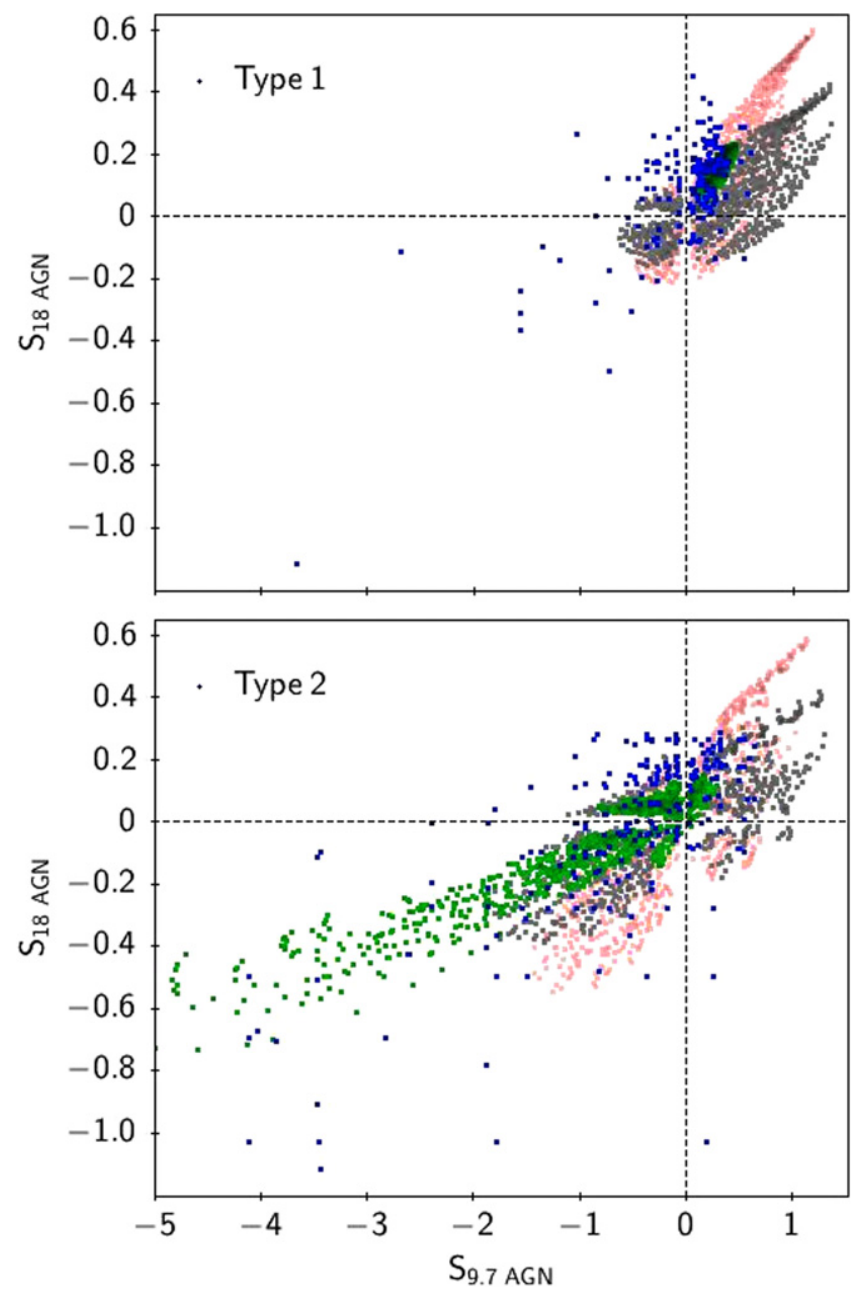

Figure 10. $S_{18 \text { AGN }}$ as a function of $S_{9.7 \mathrm{AGN}}$, overplotted on model predictions. The top (bottom) panel shows type 1 (type 2) objects (in blue). Smooth models are shown in green, and clumpy models with the Ossenkopf et al. (1992) and Draine (2003) silicates are shown in gray and pink, respectively.

Imanishi et al. (2007), a continuous dust distribution is the only morphology that can reproduce the deepest absorption features seen in the sample for type 2 views (lower left quadrant of bottom panel in Figure 10), at least without resorting to additional obscuration by the host galaxy. In fact, Levenson et al. (2007) suggested that a deep silicate absorption feature at $9.7 \mu \mathrm{m}$ requires the primary source to be embedded in a continuous, optically and geometrically thick dusty medium, while a clumpy medium with clouds illuminated from the outside will result in a much shallower feature, as the emission from these clouds will fill the absorption trough.

We visually inspected all AGNs with $S_{9.7 \text { AGN }}<-1.5$ for which we could find sufficiently high-resolution images and established that they are predominantly hosted in galaxies with high inclinations, galaxies with very visible dust lanes crossing the center (e.g., NGC 5793 or NGC 7172), or in interactive systems at various stages of their interaction (e.g., Mkr 273, Mrk 331, NGC 2623). In fact, Goulding et al. (2012) reached this same conclusion in their study of 20 nearby $(z<0.05)$ bona fide Compton-thick AGNs. This implies that the source of the deepest silicate absorption features is dust in the host galaxy rather than dust in the torus (see also Deo et al. 2007). In favor of this view, Lagos et al. (2011) find that type 1 AGNs have a strong preference in residing in face-on galaxies, while type 2 


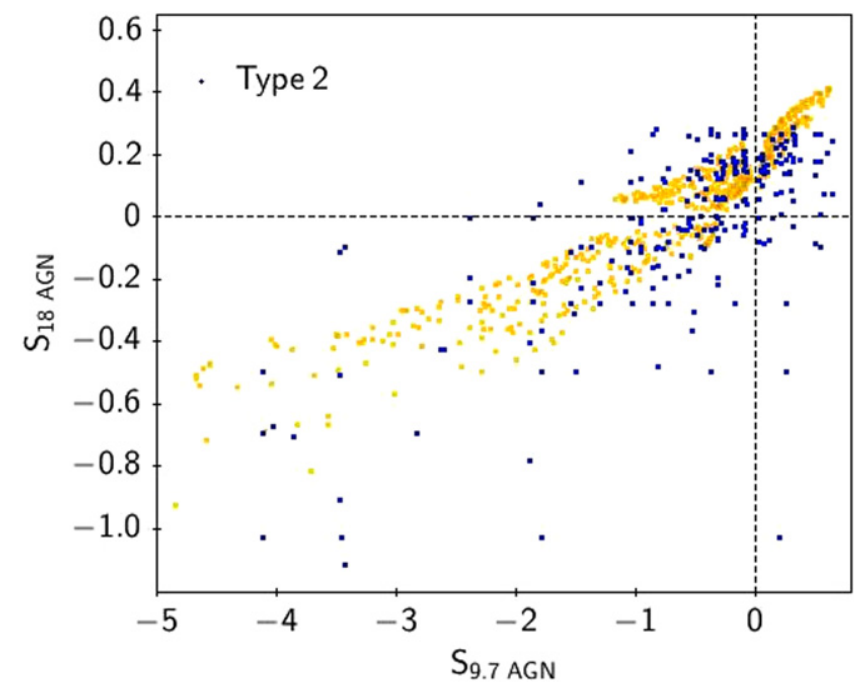

Figure 11. $S_{18 \mathrm{AGN}}$ as a function of $S_{9.7 \mathrm{AGN}}$ for type 2 objects (in blue) overplotted on smooth model predictions (in yellow) using the Feltre et al. (2012) primary source.

AGNs reside in hosts of any orientation. The deep absorption features are therefore of no relevance to the modeling of the torus and they should not be used to favor smooth dust distributions over clumpy ones. We note that this is by no means an argument against the AGN unification scheme, but does suggest that the obscuration of the nucleus, i.e., a type 2 view, may, in some cases, be decoupled from the orientation of the torus.

Finally, what is not reproduced by any of the torus models are many of the obscured and unobscured AGNs with silicate absorption at $9.7 \mu \mathrm{m}$ but emission at $18 \mu \mathrm{m}$. Smooth models do not predict such behavior in type 1 views at all, but both morphologies produce $S_{18 \text { AGN }}$ of about half the strength measured for the spectra when $S_{9.7 \mathrm{AGN}}$ is in absorption. Feltre et al. (2012) showed that the adopted primary source can also affect the properties of the silicate feature. As a last resort, and since we have no control of the primary source of the Nenkova models, we produced an additional grid of smooth models with the Ossenkopf et al. (1992) silicates but using the primary source from Feltre et al. (2012), which allows for more intrinsic AGN emission at wavelengths beyond $1 \mu \mathrm{m}$ compared to the primary source used by the Nenkova et al. (2008) models, which we have adopted for other grids. These models still fail to reproduce the objects in the upper left quadrant of the figure for type 1 views; however, they do reproduce a much larger fraction of the objects in that same quadrant for type 2 views than any of the other model grids presented before, as shown in Figure 11.

To summarize, even though the emerging picture is still somewhat confusing, things are starting to clear up: the "cosmic" silicates (Ossenkopf et al. 1992) satisfactorily represent the absorption and scattering properties of the silicates in the obscuring torus. Clumpiness is needed in order to produce absorption features in unobscured AGNs if no foreground absorber is invoked. Clumpiness can also cause the silicates to be in absorption at $9.7 \mu \mathrm{m}$ and in emission at $18 \mu \mathrm{m}$ in type 1 sources, but a primary source with more intrinsic AGN emission at $\lambda>1.0 \mu \mathrm{m}$ might be necessary to create stronger $S_{18 \text { AGN }}$ in emission when $S_{9.7 \text { AGN }}<0.0$.
Table 3

Matched Smooth and Clumpy Model Parameters

\begin{tabular}{|c|c|c|}
\hline & Smooth & Clumpy \\
\hline$Y$ & $10,30,60,100,150$ & $10,30,60,100,150$ \\
\hline$q$ & 0,1 & 0,1 \\
\hline$\gamma$ & $1.65,2.25,3.24,5.06,9.0$ & 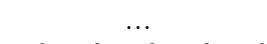 \\
\hline$\sigma$ & $\ldots$ & $70^{\circ}, 60^{\circ}, 50^{\circ}, 40^{\circ}, 30^{\circ}$ \\
\hline$\tau_{9.7}$ & $0.21,0.42,0.63,0.84,1.05$ & $\cdots$ \\
\hline$\cdots$ & $1.26,1.47,1.68,1.89,2.1$ & $\cdots$ \\
\hline$\cdots$ & $2.52,2.94,3.36,3.78,4.2$, & $\ldots$ \\
\hline$\cdots$ & $5.04,5.88,6.3,6.72,7.56$, & $\cdots$ \\
\hline$\cdots$ & $8.4,10.08,11.34,11.76,12.6$ & $\cdots$ \\
\hline$N_{0}$ & $\cdots$ & $1-5$ \\
\hline$\pi_{V}$ & $\cdots$ & $5,10,20,30,40$ \\
\hline$\cdots$ & $\cdots$ & $60,80,100,150$ \\
\hline
\end{tabular}

CASSIS is a product of the Infrared Science Center at Cornell University, supported by NASA and JPL. We made use of the NASA/IPAC Extragalactic Database (http://ned. ipac.caltech.edu). We used the TOPCAT software written by Mark B. Taylor (http://star.bris.ac.uk/mbt/topcat/). A.H.C. acknowledges funding from the Spanish Ministry of Economy and Competitiveness under grant AYA2012-31447, which is partly funded by the FEDER Program. A.F. acknowledges support from the ERC via an Advanced Grant under grant Agreement No. 321323-NEOGAL.

\section{APPENDIX \\ SMOOTH AND CLUMPY MODEL PARAMETERS}

The two original smooth and clumpy model grids cover a large but not entirely overlapping parameter space. For the purposes of this work, we matched the two sets of parameters to produce models that can be directly comparable. Both models share the inner-to-outer radius ratio, $Y$. The radial distribution of the dust or the clumps, $q$, can also be considered equivalent between the two models. For smooth models, the dust distribution in polar coordinates is given by

$$
\rho(r, \theta)=\rho_{0} \cdot r^{-q} \cdot e^{-\gamma \times|\cos (\theta)|}
$$

while clumpy models assume a Gaussian angular distribution of clouds of width $\sigma$ given by

$$
N_{T}(\beta)=N_{0} e^{\left(-\beta^{2} / \sigma^{2}\right)},
$$

where $N_{0}$ is the average number of clouds along a given radial direction on the equatorial plain and $\beta(=90-\theta)$ is the angle with respect to the axis of the torus. Considering the two extreme viewing angles for both models, the torus opening angle is of no relevance and $\gamma$ and $\sigma$ can be taken so as to match each other. While smooth models use the equatorial optical depth at $9.7 \mu \mathrm{m}, \tau_{9.7}$, as an input parameter, clumpy models use the optical depth of a single cloud calculated in the $V$-band at $0.55 \mu \mathrm{m}, \tau_{V} . \tau_{9.7}$ for clumpy models can be derived via the relation $\tau_{9.7}=0.042 \times \tau_{V} \times N_{0}$ (see Nenkova et al. 2008). Table 3 summarizes the values of each of the parameters for the matched model grids. 


\section{REFERENCES}

Antonucci, R. 1993, ARA\&A, 31, 473

Buchanan, C. L., Gallimore, J. F., O’Dea, C. P., et al. 2006, AJ, 132, 401

Clavel, J., Schulz, B., Altieri, B., et al. 2000, A\&A, 357, 839

Deo, R. P., Crenshaw, D. M., Kraemer, S. B., et al. 2007, ApJ, 671, 124

Draine, B. T. 2003, ApJ, 598, 1017

Feltre, A., Hatziminaoglou, E., Fritz, J., \& Franceschini, A. 2012, MNRAS, 426, 120

Fritz, J., Franceschini, A., \& Hatziminaoglou, E. 2006, MNRAS, 366, 767

Goulding, A. D., Alexander, D. M., Bauer, F. E., et al. 2012, ApJ, 755, 5

Granato, G. L., \& Danese, L. 1994, MNRAS, 268, 235

Hao, L., Spoon, H. W. W., Sloan, G. C., et al. 2005, ApJL, 625, L75

Hao, L., Weedman, D. W., Spoon, H. W. W., et al. 2007, ApJL, 655, L77

Hatziminaoglou, E., Fritz, J., \& Jarrett, T. 2009, MNRAS, 399, 1206

Hatziminaoglou, E., Pérez-Fournon, I., Polletta, M., et al. 2005, AJ, 129, 1198

Hernán-Caballero, A., Alonso-Herrero, A., Hatziminaoglou, E., et al. 2015, ApJ, 803, 109

Hernán-Caballero, A. 2012, MNRAS, 427, 816

Hernán-Caballero, A., \& Hatziminaoglou, E. 2011, MNRAS, 414, 500

Hönig, S. F., Beckert, T., Ohnaka, K., et al. 2006, A\&A, 452, 459

Horst, H., Gandhi, P., Smette, A., \& Duschl, W. J. 2008, A\&A, 479, 389

Houck, J. R., Roellig, T. L., van Cleve, J., et al. 2004, ApJS, 154, 18

Imanishi, M., Dudley, C. C., Maiolino, R., et al. 2007, ApJS, 171, 72

Lagos, C., Padilla, N. D., Strauss, M. A., Cora, S. A., \& Hao, L. 2011, MNRAS, 414, 2148

Lebouteiller, V., Barry, D. J., Spoon, H. W. W., et al. 2011, ApJS, 196, 8

Levenson, N. A., Sirocky, M. M., Hao, L., et al. 2007, ApJL, 654, L45

Li, M. P., Shi, Q. J., \& Li, A. 2008, MNRAS, 391, L49
Lutz, D., Maiolino, R., Spoon, H. W. W., \& Moorwood, A. F. M. 2004, A\&A, 418, 465

Maiolino, R., Shemmer, O., Imanishi, M., et al. 2007, A\&A, 468, 979

Markwick-Kemper, F., Gallager, S. C., Hines, D. C., \& Bouwman, J. 2007, ApJL, 668, L107

Mason, R. E., Levenson, N. A., Shi, Y., et al. 2009, ApJL, 693, L136

Mateos, S., Carrera, F. J., Alonso-Herrero, A., et al. 2015, MNRAS, in press Nenkova, M., Sirocky, M. M., Ivezić, Ž., \& Elitzur, M. 2008, ApJ, 685, 147 Nikutta, R., Elitzur, M., \& Lacy, M. 2009, ApJ, 707, 1550

Noll, S., Burgarella, D., Giovannoli, E., et al. 2009, A\&A, 507, 1793

Ossenkopf, V., Henning, T., \& Mathis, J. S. 1992, A\&A, 261, 567

Pier, E. A., \& Krolik, J. H. 1992, ApJ, 401, 99

Prieto, M. A., Reunanen, J., Tristram, K. R. W., et al. 2010, MNRAS, 402, 724

Schweitzer, M., Groves, B., Netzer, H., et al. 2008, ApJ, 679, 101

Shen, Y., Richards, G. T., Strauss, M. A., et al. 2011, ApJS, 194, 45

Shi, Y., Rieke, G. H., Hines, D. C., et al. 2006, ApJ, 653, 127

Shi, Y., Rieke, G. H., Ogle, P. M., Su, K. Y. L., \& Balog, Z. 2014, ApJS, 214, 23

Siebenmorgen, R., Haas, M., Krügel, E., \& Schulz, B. 2005, A\&A, 436, L5

Sirocky, M. M., Levenson, N. A., Elitzur, M., Spoon, H. W. W., \& Armus, L. 2008, ApJ, 678, 729

Smith, H. A., Li, A., Li, M. P., et al. 2010, ApJ, 716, 490

Spoon, H. W. W., Marshall, J. A., Houck, J. R., et al. 2007, ApJL, 654, L49

Sturm, E., Hasinger, G., Lehmann, I., et al. 2006, ApJ, 642, 81

Sturm, E., Schweitzer, M., Lutz, D., et al. 2005, ApJL, 629, L21

Thompson, G. D., Levenson, N. A., Uddin, S. A., \& Sirocky, M. M. 2009, ApJ, 697, 182

Wu, Y., Charmandaris, V., Huang, J., Spinoglio, L., \& Tommasin, S. 2009, ApJ, 701, 658 